\title{
Proprotein convertases modulate budding and branching morphogenesis of rat ventral prostate
}

\author{
KATSUNORI UCHIDA ${ }^{1,2}$, MASAHIRO KANAI ${ }^{1}$, SHIGENORI YONEMURA ${ }^{1}$, KENICHIRO ISHII ${ }^{1}$, \\ YOSHIFUMI HIROKAWA ${ }^{2}$ and YOSHIKI SUGIMURA ${ }^{1, *}$ \\ ${ }^{1}$ Nephro-Urologic Surgery and Andrology, Division of Reparative and Regenerative Medicine and ${ }^{2}$ Pathologic Oncology, Division of \\ Molecular and Experimental Medicine, Mie University Graduate School of Medicine, Mie, Japan
}

\begin{abstract}
The onset of prostate morphogenesis is involved in the interaction between mesenchyme and epithelium. Proprotein convertases (PCs) activate a variety of growth and differentiation factors including mesenchymal and epithelial factors, such as insulin-like growth factor (IGF) and transforming growth factor- $\beta$ (TGF- $\beta$ ), which induce ductal budding and branching. In this study, we provide evidence that PCs play a critical role in prostatic budding from the urogenital sinus (UGS) and ductal branching morphogenesis of the neonatal rat ventral prostate. PCs were expressed only in the epithelial cells of neonatal rat prostate. PC activity in the ventral prostate was modulated by endogenous androgen. PC inhibition suppressed prostatic budding and branching. Taken together, our data indicates that androgen-induced PCs initiate the development of the prostate.
\end{abstract}

KEY WORDS: proprotein convertase, prostate, development, furin, PC1

Proprotein convertases (PCs) have been known as processing proteases that convert a wide variety of precursor proteins to their mature active form. To date, eight PCs have been identified including furin, PC1/3, PC2, PC4, PACE4, PC5/6 (and its isoform PC5B), PC7/ 8 and $\mathrm{SKI}-1$. All PCs except for SKI-1 cleave precursor proteins at basic residues within the consensus motif $(K / R)-(X)_{n}-(K / R) \downarrow$, when $\mathrm{n}=0,2,4$ or 6 and $\mathrm{X}$ is usually not Cys. All PCs except for SKI-1 are responsible for processing of matrix metalloproteinases (MMPs), growth factors, membrane receptors and adhesion molecules (Khatib etal., 2004). Therefore, these processing enzymes are suggested to have a fundamental role in embryogenesis, development and disease via the activation of their substrates (Thomas 2002).

The prostate is a male sex accessory gland and develops from the embryonic urogenital sinus (UGS). Prostatic buds emerge from urogenital sinus epithelium (UGE) and extend into urogenital sinus mesenchyme (UGM) where they undergo ductal branching morphogenesis under androgenic stimulation of interactions between mesenchymal and epithelial cells (Sugimura et al., 1986). In the developing prostate, both growth and differentiation factors are derived from mesenchyme via androgenic stimulation which induce prostatic epithelial growth (Hayward et al., 2000). As candidates of these factors, insulin-like growth factor (IGF-1), hepatocyte growth factor (HGF), transforming growth factor- $\beta$ (TGF- $\beta$ ), bone morphogenetic protein-4 (BMP-4) and prostate differentiation factor (PDF) were reported previously (Hayward et al., 2000, Lamm et al., 2001, Paralkar etal., 1998). Many growth and differentiation factors are first synthesized as larger biologically inactive precursors, which are proteolytically activated by PCs. Previously we reported that the inhibition of PC activity resulted in the loss of the luminal cell phenotype and induction of the basal cell phenotype in LNCaP (Uchida et al., 2003). Our results suggest that PCs activity may be involved in the regulation of prostate epithelial cell differentiation. The role of PCs in the development of the prostate remains largely unknown. Therefore, we hypothesized that PCs regulate budding and branching morphogenesis of the prostate epithelium via activation of growth and differentiation factors.

In this study, we investigated the distribution of PCs and the effect of the PCs inhibitor, decanoyl-Arg-Val-Lys-Arg-chloromethylketone (CMK) on prostatic bud formation from the UGS and the development of rat ventral prostate.

\section{Results and Discussion}

\section{PC expression in the UGS and neonatal rat prostate}

All PCs except for PC4, which is confined to the testis and ovary, are expressed widely in a variety of tissues and cells (Hatsuzawa et al., 1990). However, the expression, localization

Abbreviations used in this paper: CMK, decanoyl-Arg-Val-Lys-Argchloromethylketone; IGF, insulin-like growth factor; PC, proprotein convertase; TGF, transforming growth factor; UGS urogenital sinus; VP, ventral prostate.

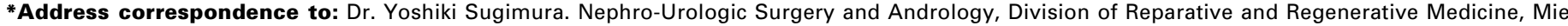
University Graduate School of Medicine, 2-174 Edobashi, Tsu, Mie, 514-8507 Japan. Fax: +81-59-231-5203. e-mail: sugimura@clin.medic.mie-u.ac.jp
} 


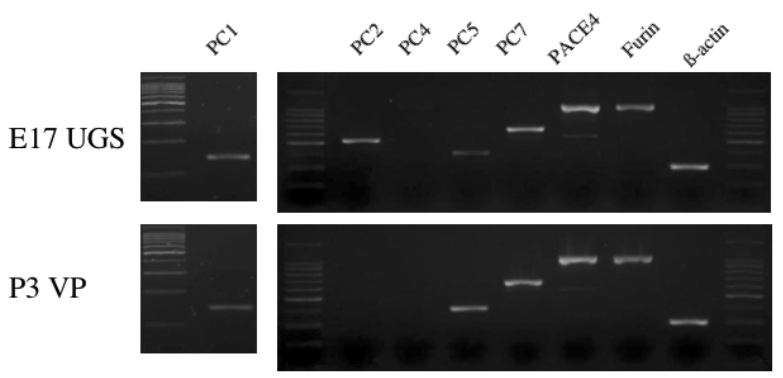

Fig. 1. Proprotein convertase (PC) mRNA expression in E17 urogenital sinus (UGS) and P3 ventral prostate. Amplification products for PC1 and other PCs were separated by $2 \%$ and $4 \%$ agarose gel electrophoresis, respectively. PC2 mRNA was detected in the E17 UGS, not in the P3 ventral prostate

Fig. 2. PC1 distribution in the developing prostate. Light micrographs of $H E$ staining of $P 3$ rat prostate $(\mathbf{A}, \mathbf{C})$ and immunostaining for $P C 1$ (B,D). PC1-positive cells were detected in the epithelial cells of the VP (B), SV and VD (D). PC1 staining was not observed in the epithelial cells of the urethra and mesenchymal cells of the prostate. Abbreviations: SV, seminal vesicle; UR urethra; VD, vas deferens; VP, ventral prostate. Bar, $200 \mu \mathrm{m}$.

and function of PCs in the prostate are still unknown. We first examined the gene expression and protein distribution of PCs in the developing prostate. Gene expressions of all PCs except for PC4 were detected in E17 UGS by RT-PCR. In contrast, PC2 and PC4 gene expression was not detected in P3 VP, whereas mRNAs of PC1, PC5, PC7, furin and PACE4 were observed (Fig. 1). Next we confirmed PCs expression and distribution by immunofluorescence staining. We examined the distribution of furin and PC1 in the developing prostate by immunofluorescence staining. At P3, cytoplasmic staining of PC1 was observed in the epithelial cells of the prostate, seminal vesicle and vas deferens. PC1 immunoreactivity was not observed in the mesenchymal cells of the prostate (Fig. 2). PC1 was also detected in endothelial cells and red blood cells. The distribution of furin-positive cells was similar to that of PC1-positive cells (data not shown). Furin and $\mathrm{PC} 1$ expressions were not observed in urethral mesenchyme and epithelium. At E17, furin and PC1 were detected in the epithelial cells of the vas deferens, but not epithelial cells of the UGS (data not shown). The discrepancy in PCs expression at UGS between RT-PCR and immunofluorescence staining could be due to the lower expression of PCs or the contamination of the vas deferens.

\section{The modulation of PC activity in the neonatal ventral prostate}

Previous studies demonstrated that androgenic effects on epithelial growth, budding and ductal branching morphogenesis of the prostate were mediated by mesenchymal androgen receptors (Cunha et al., 1980). Therefore, we hypothesized that the activity of epithelial PCs could be regulated by testosterone via epithelial-mesenchymal interaction. To investigate regulation of PCs activity by testosterone, E17 UGS and P1 VPs were cultured with or without testosterone. Comparable cultures were treated with $\mathrm{CMK}$, the PC inhibitor. Results demonstrated that PCs activity was significantly increased by the addition of testosterone and $\mathrm{PCs}$ activity induced by testosterone was significantly inhibited by CMK at $50 \mu \mathrm{M}$ in P1 VP (Fig. 3B), but not in E17 UGS (Fig. 3A).
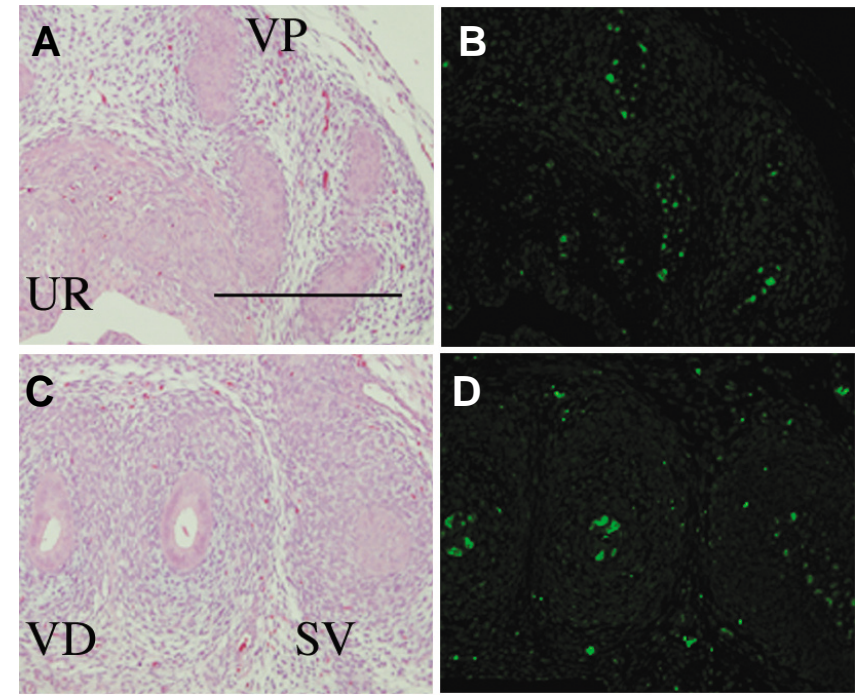

Our results suggested that PC were expressed in the epithelium of the prostate during the period of budding and branching morphogenesis and that the activity of epithelial PCs was regulated by testosterone via epithelial-mesenchymal interaction. Our results also showed that testosterone treatment had minimal effect on P1 VP and did not affect PCs activity in UGS. These could be due to the lower activity of specific PC modulated by

A

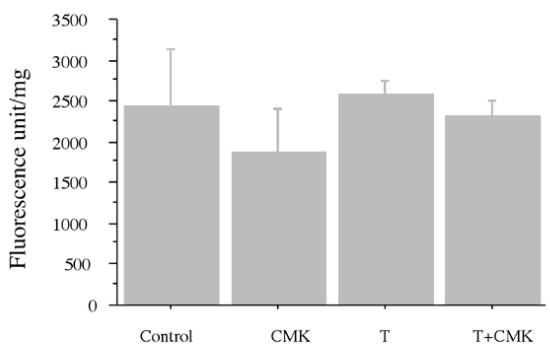

B

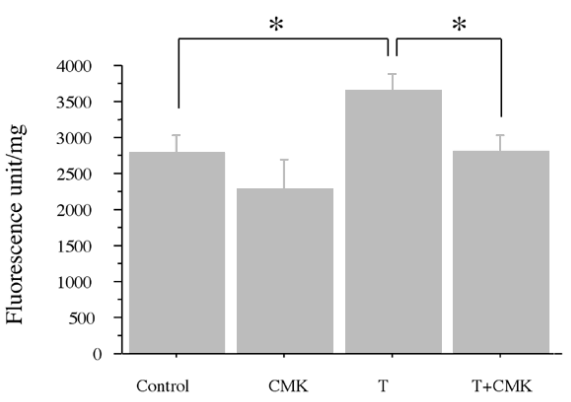

Fig. 3. Proprotein convertase (PC) activity as measured by bocRVRR-AMC processing. (A) The effect of $10 \mathrm{nM}$ testosterone and 50 $\mu M C M K$ on PC activity in the urogenital sinus. No significant differences were observed. (B) The effect of $10 \mathrm{nM}$ testosterone and $50 \mu \mathrm{M} \mathrm{CMK}$ on $P C$ activity of the rat $P 1$ ventral prostate. $P C$ activity was significantly increased by the addition of $10 \mathrm{nM}$ testosterone. Testosterone-induced $P C$ activity was significantly decreased by $50 \mu M C M K .{ }^{*} p<0.05$ 


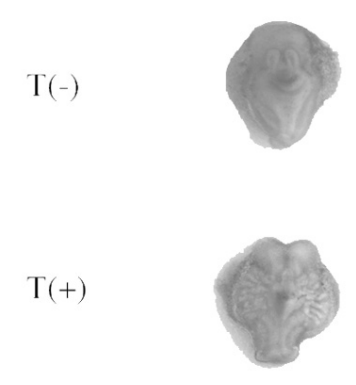

$\mathrm{CMK}(\mu \mathrm{mol} / \mathrm{l})$
0

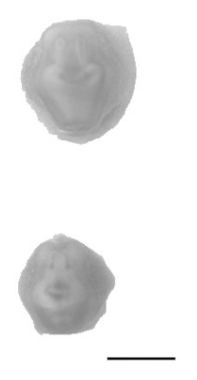

50
A
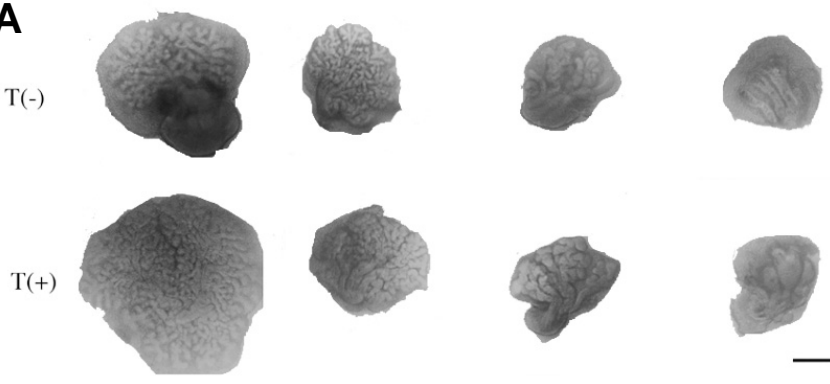

$\mathrm{CMK}(\mu \mathrm{mol} / \mathrm{l}) \quad 0$

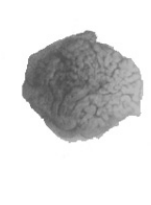

1

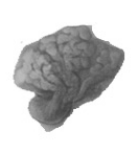

10

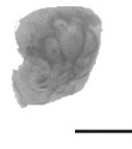

50
Fig. 4. Effect of the proprotein convertase inhibitor, CMK, on prostatic bud formation. Representative images of rat urogenital sinus (UGS) cultured for 4 days in serum free medium without testosterone and CMK, with $10 \mathrm{nM}$ testosterone alone, with both $50 \mu \mathrm{M} C M \mathrm{~K}+10 \mathrm{nM}$ testosterone and $50 \mu \mathrm{M} C \mathrm{MK}$ alone. Prostatic budding was observed by androgen stimulation. Androgen-stimulated budding was inhibited by treatment with $50 \mu \mathrm{M} \mathrm{CMK}$. Bar, $1 \mathrm{~mm}$.

testosterone or the selectivity of boc-RVRR-AMC for specific PC, because wide variety of PCs was expressed in VP and UGS.

Effect of the proprotein convertase inhibitor, CMK, on prostatic bud formation and branching morphogenesis

We next focused the effect of PCs on the prostatic budding and branching morphogenesis using in vitroorgan culture system. Rat E17 UGS were cultured for 4 days and P1 VPs for 6 days. In the presence of testosterone at $10 \mathrm{nM}$, prostatic buds emerged from the UGE and elongated into the UGM. Testosterone-induced prostatic budding was inhibited by the addition of $50 \mu \mathrm{M} \mathrm{CMK}$ (Fig. 4). Neonatal VPs grew and underwent ductal brunching morphogenesis extensively with $10 \mathrm{nM}$ testosterone. The addition of CMK inhibited the growth of neonatal prostate and significantly induced the reduction of the number of ductal tips with or without $10 \mathrm{nM}$ testosterone in a dose- and a time-dependent manner (Fig 5A, B).

Histological and immunohistochemical analysis of cultured VPs was examined. Microscopically, the higher frequency of canalization was observed in the prostate treated with testosterone alone, not in the prostate with CMK (Fig. 6A). The diameter of ducts was significantly increased by the addition of CMK (Fig. $6 \mathrm{~B})$. In the prostate treated with testosterone alone, nuclear

TABLE 1

\section{PRIMERS USED FOR RT-PCR AMPLIFICATION}

\begin{tabular}{llcc} 
Gene & Primers & fragment size (bp) & Reference \\
\hline PC1/3 & S': 5'-GCTACTAATCTCACTCAAAGC-3' & 143 & Lee 1999 \\
& AS: 5'-CCTTCTCTTAATATGCCAAC-3' & & \\
PC2 & S: 5'-GCTAGACTTGAATGTGGC-3' & 525 & Rovere 1996 \\
& AS: 5'-GTTGCAGTCATCGTAGCT-3' & & \\
PC4 & S: 5'-AGCCGCAACACATACACATC-3' & 680 & \\
& AS: 5'-GCCATCGCAGCATACAGTCA-3' & & Akamatsu 2000 \\
PC5 & S: 5'-CTGCTGGTTTAAAGGTGAGCCA-3' & 403 & \\
& AS: 5'-TCACCAGCAGCTCTTTCTCC & & \\
PC7 & S: 5'-GCCCAGGAAGAGACATCAAT-3' & 640 & \\
& AS: 5'-CATCTTGTCTCCTCCACTGA-3' & & \\
PACE4 & S: 5'-CCCTCTGGAACCAAGTCTCAACTT-3' & \multirow{2}{*}{1041} & \\
& AS: 5'-TGAAGCCAGCTTTACATCTGCTGC-3' & & \\
Furin & S: 5'-AATGGTGTCTGTGGTGTAGG-3' & 1050 & \\
& AS: 5'-GTCAGCGTCCCATAGTTGTT-3' & & \\
\hline
\end{tabular}

aS, sense: AS, antisense
B

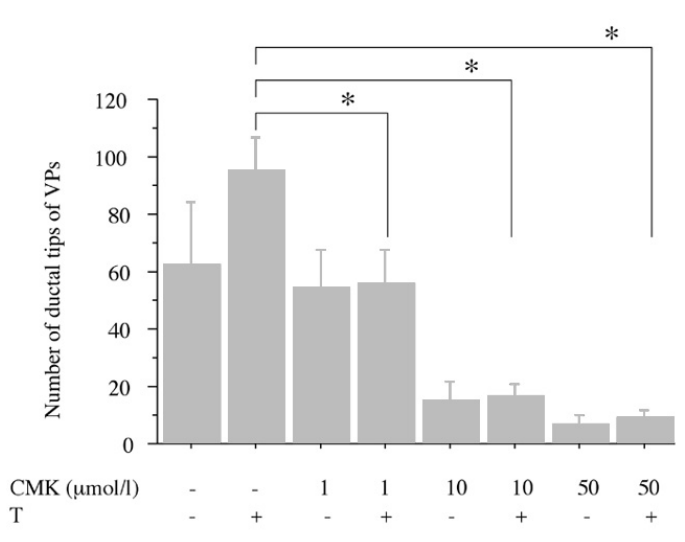

Fig. 5. Effect of the proprotein convertase inhibitor, CMK, on branching morphogenesis. (A) Representative images of rat $P 1$ ventral prostates cultured for 6 days in serum free medium with/without $10 \mathrm{nM}$ testosterone, with various concentrations of CMK. Prostatic ductal growth and branching were observed by androgen stimulation. Androgen-stimulated growth was inhibited by treatment with CMK in a dosedependent manner. Bar, $2 \mathrm{~mm}$. (B) Quantification of branching with CMK treatment. Branching was significantly increased with testosterone and significantly inhibited by the addition of CMK in a dose-dependent manner. ${ }^{*} p<0.05$

staining of PCNA was more frequently observed in the ductal tip than in the base. of the prostate by immunohistochemical staining. In contrast, PCNA expression was frequently observed in the tip and base of the prostate treated with testosterone + CMK (Fig. 6C).

Prostatic development is characterized by the bud formation, canalization and branching mediated via the epithelial-mesenchymal interaction under the balance of stimulatory and inhibitory factors. Among PCs substrates, IGF-1 and HGF are considered to be responsible for prostatic development as the stimulatory factors and BMP-4 is known as the inhibitory factor. TGF- $\beta$ has the stimulatory effects on the periphery and inhibitory effects on the center of the prostate (Tomlinson et al., 2004). In addition, previous study demonstrated that MMPs and integrins, which were considered as PCs substrates, also had the stimulatory effect on the branching of mammary gland and ureteric bud (Simian et al., 2001, Pohl et al., 2000, Zent et al., 2001). PCs inhibition might cause an imbalance in the activation of stimulatory and inhibitory substrates. In fact, our macroscopic observa- 
A

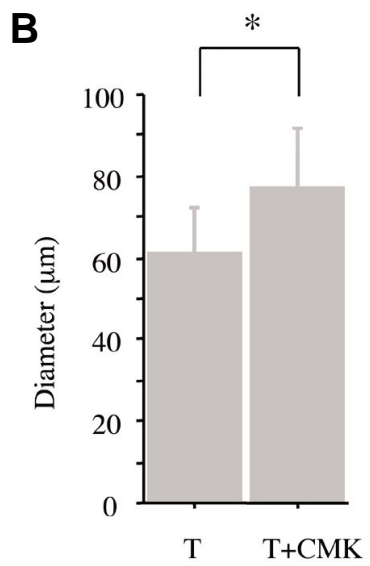

C

Tip

Base

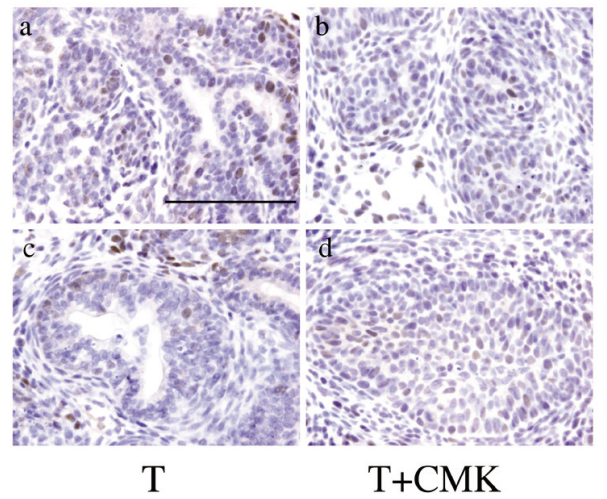

Fig. 6. Effect of the proprotein convertase inhibitor, CMK, on branching morphogenesis - microscopic and immunohistochemical analysis. (A) Representative microscopic images (HE staining) of rat $P 1$ ventral prostates cultured for 6 days in serum free medium with $10 \mathrm{nM}$ testosterone, with/without $10 \mu \mathrm{M} \mathrm{CMK}$. The higher frequency of canalization, the lower cell density of epithelium was observed in the prostate treated with testosterone without CMK (a), not in the prostate with CMK (b). Bar, 200 $\mu \mathrm{m}$. (B) The diameter of ducts with testosterone, with/without CMK. The diameter was significantly increased by the addition of CMK. ${ }^{*} p<0.05$. (C) Immunostaining for PCNA of the prostate treated with testosterone alone $(a, c)$ and with testosterone and $C M K(b, d)$. PCNA expression was observed in the ductal tip (a, b) and base (c, d). Bar, $200 \mu \mathrm{m}$.

tion showed that PCs inhibitor reduced prostatic budding and branching, suggesting that BMP-4 activation might be accelerated by the inhibition of PCs. On the other hand, microscopic observation showed that PCs inhibitor induced the enlargement of the prostatic epithelial cord and the reduction of canalization. Thease results could be associated with the inactivation of morphogens, such as TGF- $\beta$ and PDF, by the inhibition of PCs. In addition, PCNA expression indicated that PCs inhibitor initiated the epithelial proliferation in the base, which might represent the imbalance of the prostatic growth and be resulted in the enlargement of the prostatic epithelial cord. Our results indicate PCs are involved in the usual process of the prostatic development, including the budding, cord formation, canalization and branching. Therefore, the difference of the phenotypic expression in the prostate treated with or without PCs inhibitor would be important.
The expression and localization of each PC, by which substrates are predominantly activated and responsible for the each steps of prostatic development, should be studied further.

In conclusion, our results suggest an important role of PCs in the prostatic morphogenesis. PCs modulated by androgen are expressed in neonatal VP and may be responsible for the processing of mesenchymal and epithelial factors, which initiate budding and branching of the prostate.

\section{Materials and Methods}

\section{Materials}

The animal use procedures were in accordance with the guide for the care and use of laboratory animals of Mie University Graduate School of Medicine. Sprague-Dawley (SD) rats were obtained from CLEA (Tokyo, Japan). Testosterone, insulin and transferrin were obtained from Sigma at MO. DMEM-Ham's F12 was purchased from Invitrogen (Carlsbad, CA), gentamycin from Wako (Osaka, Japan). N-t-butoxycarbonyl-Arg-ValArg-Arg-7-amino-4-methylcoumarine (Boc-RVRR-AMC) was obtained from Bachem (King of Prussia, PA) and decanoyl-Arg-Val-Lys-Argchloromethylketone (CMK) from Sigma (St. Louis, MO). Anti-furin and anti-PCNA antibodies were purchased from Santa Cruz (Santa Cruz, CA). Anti-PC1 antiserum was kindly gifted by Dr. Tanaka at Shizuoka University in Japan. FITC-labeled secondary antibody was purchased from MBL (Nagoya, Japan).

\section{Tissue collection}

Rat UGS and VPs were obtained from SD rats. The day of detection of a vaginal plaque and the day of birth was designed E0 and P0, respectively. UGS from E17 rats and VPs from P0 and P3 neonatal rats were dissected and used for immunofluorescence staining, PC activity assay and in vitro organ culture.

\section{RT-PCR Analysis}

Total RNA was prepared from E17 UGS and P3 VP using RNeasy (Qiagen, CA). cDNA was prepared using Superscript (Invitrogen, CA). The efficiency of each cDNA reaction was assayed by amplification of $\beta$ actin transcripts with primers for $\beta$-actin (R \& D systems, MN). Primers used to amplify PCs gene were shown in Table1. The viability of the primer for PC4 was conformed using neonatal rat testis. The first strand CDNA was amplified with amplitaq gold (Applied biosystems, NJ). The PCR condition was as follows: 1 cycle at $95^{\circ} \mathrm{C}$ at for $5 \mathrm{~min} ; 35$ cycles at $95^{\circ} \mathrm{C}$ for $15 \mathrm{~s}, 53-57^{\circ} \mathrm{C}$ for $30 \mathrm{~s}, 72^{\circ} \mathrm{C}$ for $1 \mathrm{~min} 30 \mathrm{~s}$. Amplification products $(5$ $\mu \mathrm{l})$ were separated by $2-4 \%$ agarose gel electrophoresis.

\section{Immunofluorescence staining}

Dissected E17 UGS and P3 prostate were fixed with 10\% formaldehyde for $3 \mathrm{~h}$ on ice, processed into paraffin and then sectioned at $3 \mu \mathrm{m}$. Sections were deparaffinized with Histoclear. To enhance immunoreactivity for PCs, sections were oxidized before immunostaining with Gomori's oxidation mixture, as described previously (Kurabuchi and Tanaka, 1997). Rat pancreas was used as the positive control. Nonspecific binding was blocked with blocking buffer (DAKO, CA) for 1 hour at room temperature. Sections were sequentially incubated overnight at $4^{\circ} \mathrm{C}$ with following reagents: anti-furin antibody (1:50), anti-PC1 antiserum (1:500). The next day, sections were rinsed and incubated in a FITC-labeled secondary antibody and processed according to the manufacture's instructions.

\section{Proprotein convertase activity assay}

E17 UGS and P1 VP were cultured with and without $10 \mathrm{nM}$ testosterone and $50 \mu \mathrm{M} \mathrm{CMK}$. After $24 \mathrm{~h}$ incubation, UGS and VP were washed with PBS and incubated with assay medium which consisted of the growth medium containing $0.25 \%$ Triton X-100 to permeabilize cells and bocRVRR-AMC $(100 \mu \mathrm{M})$ as a fluorogenic substrate. Fluorescence was 
measured at $360 \mathrm{~nm}$ excitation and $460 \mathrm{~nm}$ emission wavelengths after $4 \mathrm{~h}$ of substrate addition. Tissue weights of UGS and VPs were measured for normalization before the addition of assay medium. The data were normalized to tissue weights

\section{In vitro organ culture}

UGS from E17 rats and VPs from P1 rats were grown in an organ culture system as previously described with modifications (Sugimura et al., 1996). Briefly, dissected tissues were placed on Millicell membranes (Millipore, Bedford, MA) in 6-well plates (BD bioscience, Franklin Lakes, $\mathrm{NJ}$ ) and cultured in DMEM-Ham's F12 without phenol red supplemented with insulin $(10 \mu \mathrm{g} / \mathrm{ml})$, transferrin $(10 \mu \mathrm{g} / \mathrm{ml})$ and gentamycin $(50 \mu \mathrm{g} / \mathrm{ml})$ at $5 \% \mathrm{CO}_{2}$. Testosterone $(10 \mathrm{nM})$ and various concentrations of $\mathrm{CMK}$ were added and the medium was changed every day. Individual ductal tips were counted manually. Four to five VPs or UGSs were used for each group in one experiment. The experiment was repeated at least three times.

\section{Histological and immunohistochemical analysis of cultured VPS}

The prostates cultured with $10 \mathrm{nM}$ testosterone with/without $10 \mu \mathrm{M}$ $\mathrm{CMK}$, were processed into paraffin and then sectioned at $3 \mu \mathrm{m}$. Sections were deparaffinized with Histoclear, used for Hematoxylin-Eosin (HE) staining and immunohistochemical staining. The diameter of the prostattic duct was measured under light microscope. Immunohistochemical staining was performed with Vectastain $\mathrm{ABC}$ kit (Vectastain Laboratories, $\mathrm{CA}$ ). Briefly, sections were incubated overnight at $4^{\circ} \mathrm{C}$ with following reagents: anti-PCNA antibody (1:5000), The next day, sections were rinsed and incubated in a secondary antibody and processed.

\section{Statistical analysis}

Data are presented as the mean SD of four to five samples in each of two to six independent trials. Statistical analysis was performed using the Student $t$ test. $\mathrm{P}$ value $<0.05$ was considered significant.

\section{Acknowledgements}

This work was supported by the Ministry of Education, Culture, Sports, Science and Technology of Japan (13671685, 14571495, 15390489), the Ministry of Health, Labor and Welfare of Japan (1700108-01) and Suzuki fundation of urology. We thank Dr. Tanaka for providing us anti-PC1 antiserum and Mrs. Hiroko Nishii for technical supports.

\section{References}

AKAMATSU, T., MATSUDA, Y., TSUMURA, K., TADA, J., PARVIN, M. N., WEI, W. KANAMORI, N. and HOSOI, K. (2000) Highly regulated expression of subtilisinlike proprotein convertase PACE4 (SPC4) during dentinogenesis. Biochem. Biophys. Res. Commun. 272, 410-415.

CUNHA, G. R., CHUNG, L. W., SHANNON, J. M. and REESE, B. A. (1980) Stromalepithelial interactions in sex differentiation. Biol. Reprod. 22, 19-42.

TOMLINSON, D. C., FREESTONE, S. H., GRACE, O. C. and THOMSON, A. A. (2004) Differential effects of transforming growth factor- $\beta 1$ on cellular proliferation in the developing prostate. Endocrinology 145, 4242-4300.

HATSUZAWA, K., HOSAKA, M., NAKAGAWA, T., NAGASE, T., SHODA, A., MURAKAMI, K. and NAKAYAMA, K. (1990) Structure and expression of mouse furin, a yeast Kex2-related protease. Lack of processing of coexpressed prorenin in GH4C1 cells. J. Biol. Chem. 265, 22075-22078.

HAYWARD, S. W. and CUNHA, G. R. (2000) The prostate: development and physiology, Radiologic Clinics of North America 38, 1-14.

KHATIB, A. M., SIEGFRIED, G., CHRETIEN, M., METRAKOS, M. and SEIDAH, N.G. (2002) Proprotein convertases in tumor progression and malignancy. Am. J. Pathol. 160,1921-1935.

KURABUCHI, S. and TANAKA S. (1997) Immunocytochemical localization of prohormone convertases $\mathrm{PC} 1$ and $\mathrm{PC} 2$ in the anuran pituitary gland: subcellular localization in corticotrope and melanotrope cells. Cell Tissue Res. 288, 485496.

LAMM, M. L. G., PODLASEK, C. A. and BARNET, D. H. (2001) Mesenchymal factor bone morphogenetic protein 4 restrict ductal budding and branching morphogenesis in the developing prostate. Dev. Biol. 232, 301-314.

LEE, Y. C., DAMHOLT, A. B., BILLESTRUP, N,. KISBYE, T., GALANTE, P., MICHELSEN, B., KOFOD, H. and NIELSEN, J. H. (1999) Developmental expression of proprotein convertase $1 / 3$ in the rat. Mol. Cel/ Endocrinology 155 , 27-35.

PARALKAR, V. M., VAIL, A. L. and GRASSER, W. A. (1998) Cloning and characterization of a novel member of the transforming growth factor- $\beta$ /bone morphogenetic protein family. J. Biol. Chem. 273,13760-13767.

POHL, M., SAKURAI, H., BUSH, K. T. and NIGAM, S. K. (2000) Matrix metalloproteinases and their inhibitors regulate in vitro ureteric bud branching morphogenesis. Am. J. Physiology-Renal Physiology 279, 891-900.

ROVERE, C., BARBERO, P. and KITABGI, P. (1996) Evidence that PC2 is the endogenous pro-neurotensis convertase in rMTC 6-23 cells and that PC1- and PC2-transfected PC12 cells differentially process pro-neurotensin. J. Biol. Chem. 271, 11368-11375

SIMIAN, M., HIRAI, Y., NAVRE, M., WERB, Z., LOCHTER, A. and BISSELL, M. J. (2001) The interplay of matrix metalloproteinases, morphogens and growth factors is necessary for branching of mammary epithelial cells. Development 128, 3117-3131.

SUGIMURA, Y., CUNHA, G. R. and DONJACOUR, A. A. (1986) Morphogenesis of ductal networks in the mouse prostate. Biol. Reprod. 34, 961-71.

SUGIMURA, Y., FOSTER, B. A., HOM, Y. K., LIPSCHUTZ, J, H., RUBIN, J. S., FINCH, P. W., AARONSON, S. A., HAYASHI, N., KAWAMURA, J. and CUNHA, G. R. (1996) Keratinocyte growth factor(KGF) can replace testosterone in the ductal branching morphogenesis of the rat ventral prostate. Int. J. Dev. Biol. 40, 941-951.

THOMAS, G. (2002) Furin at the cutting edge: from protein traffic to embryogenesis and disease. Nature Review Mol. Cell. Biol. 10, 753-66.

UCHIDA, K., CHAUDHARY, L. R., SUGIMURA, Y., ADKISSON, H. D. and HRUSKA, K. A. (2003) Proprotein convertases regulate activity of prostate epithelial cell differentiation markers and are modulated in human prostate cancer cells. J. Cell Biochem. 88, 394-399.

ZENT, R., BUSH, K. T., POHL, M. L., QUARANTA, V., KOSHIKAWA, N., WANG, Z., KREIDBERG, J. A., SAKURAI, H., STUART, R. and NIGAM, S. K. (2001). Involvement of laminin binding integrins and laminin-5 in branching morphogenesis of the ureteric bud during kidney development. Dev. Biol. 238, 289-302.

Received: 3rd January 2006 Reviewed by Referees: 12th April 2006 Modified by Authors and Accepted for Publication: 18th December 2006 Published Online: 9th February 2007 